\title{
Prediction in the face of uncertainty: A Monte Carlo-based approach for systems biology of cancer treatment
}

\author{
Christoph Wierling*, Alexander Kühn, Hendrik Hache, Andriani Daskalaki, Elisabeth Maschke-Dutz, \\ Svetlana Peycheva, Jian Li, Ralf Herwig, Hans Lehrach
}

Max Planck Institute for Molecular Genetics, Ihnestrasse 63-73, 14195 Berlin, Germany

\section{A R T I C L E I N F O}

\section{Article history:}

Received 12 January 2012

Accepted 13 January 2012

Available online 23 January 2012

\section{Keywords:}

Systems biology

Cancer

Modeling

Personalized medicine

Monte Carlo simulation

\begin{abstract}
A B S T R A C T
Cancer is known to be a complex disease and its therapy is difficult. Much information is available on molecules and pathways involved in cancer onset and progression and this data provides a valuable resource for the development of predictive computer models that can help to identify new potential drug targets or to improve therapies. Modeling cancer treatment has to take into account many cellular pathways usually leading to the construction of large mathematical models. The development of such models is complicated by the fact that relevant parameters are either completely unknown, or can at best be measured under highly artificial conditions. Here we propose an approach for constructing predictive models of such complex biological networks in the absence of accurate knowledge on parameter values, and apply this strategy to predict the effects of perturbations induced by anti-cancer drug target inhibitions on an epidermal growth factor (EGF) signaling network. The strategy is based on a Monte Carlo approach, in which the kinetic parameters are repeatedly sampled from specific probability distributions and used for multiple parallel simulations. Simulation results from different forms of the model (e.g., a model that expresses a certain mutation or mutation pattern or the treatment by a certain drug or drug combination) can be compared with the unperturbed control model and used for the prediction of the perturbation effects. This framework opens the way to experiment with complex biological networks in the computer, likely to save costs in drug development and to improve patient therapy.
\end{abstract}

(C) 2012 Elsevier B.V. All rights reserved.

\section{Introduction}

Mathematical modeling and simulation techniques are valuable tools for the understanding of complex systems in different areas of science. In recent years, this approach gained increasing popularity also in biology resulting in the establishment of the research area of systems biology [1-3]. Systems biology tries to understand biological systems by means of mathematical models, thus providing a tool to study and predict the behavior of biological systems in silico. In contrast to the situation in many areas in physics, where it has been possible to make highly accurate predictions based on a small number of assumptions, accurate predictions of biological processes depend on the behavior of complex networks of molecular, cellular and even organismal interactions, which have been shaped by events hundreds of millions of years ago. It is therefore quite likely that predictions in biology will have to be based to a large extent on the detailed knowledge of the components of the networks involved, as well as their interactions. While

\footnotetext{
* Corresponding author. Tel.: +49 308413 1272; fax: +49 3084131380 . E-mail address: wierling@molgen.mpg.de (C. Wierling).

URL: http://www.molgen.mpg.de/ sysbio/ (C. Wierling).
}

inherently difficult to achieve, any progress in our ability to predict the behavior of these biological networks can have enormous practical consequences. Improved predictions on the response of individual patients could, for example, decide between life and death of the individuals involved, while improved predictions on the effect of drugs could very well help to revolutionize drug development, and therefore have enormous economic value.

To allow such predictions, two basic strategies have been considered: the identification of statistical correlations in the therapy response of specific 'biomarkers' (e.g., transcripts, proteins, metabolites, patterns of genomic methylation), and the modeling of the disease and therapy, to represent accurately the biological processes in the individual patient. While statistical procedures have been quite successful in, e.g., predicting treatment responses, they are, however, inherently a relatively blunt instrument, only able to detect very strong correlations, which hold up across large groups, irrespective of the multiple differences between the individuals which make up these groups. Predictive models, in contrast, can take into account the individual situation in every patient, and could therefore, in many cases, provide more reliable predictions.

The development of such predictive models is however complicated by the lack of information on many of the model parameters, in particular the reaction kinetics. Information on the kinetics and 
kinetic parameters is either not available at all, or, at best, is based on experiments often carried out under conditions quite different from those in the living cell. Concentrations of many reactants are usually unknown, or it is simply not feasible to determine them for every individual patient. Thus, computational modeling approaches must primarily face the challenge of coping with this lack of information to come up with qualitative or even quantitative predictions of the biological system that can help to understand diseases such as cancer much better.

Clinical as well as basic research suggests that cancer is the result of an accumulation of many factors that promote tumor growth and metastasis $[4,5]$. Cancer, like many other diseases, is caused by disturbances in the complex interaction networks of biological processes in the organism. Diagnosis and therapy of these diseases require a detailed understanding of theses processes in health and disease. Due to the complexity of the problem it can only be achieved with a systems biology approach and the development of appropriate mathematical models and modeling approaches can support the formulation of new hypotheses significantly.

However, quantitative modeling efforts, e.g., using systems of ordinary differential equations (ODEs), are often hampered by the lack of detailed information about reaction kinetics and their associated parameters. An alternative to quantitative approaches is qualitative modeling, such as Boolean models or other discrete modeling approaches, however their predictive power is limited to qualitative results. Using quantitative models based on ODEs instead, requires often appropriate assumptions about the kinetic parameters.

In this article we propose an approach for predicting the effects of disturbances induced by targeted cancer therapies on the EGF signaling network. We introduce a Monte Carlo type strategy incorporating repeated simulations with parameter vectors sampled from a given random distribution and subsequent statistical significance testing, and demonstrate the applicability of the approach by generating statistically reproducible predictions of potential drug targets.

\section{EGF signaling network and associated targeted cancer therapies}

Epidermal growth factor (EGF) mediated signaling, like other receptor tyrosine kinase signaling pathways, is known to be involved in the regulation of cell proliferation and survival [6]. In humans, more than 30 ligands and four receptors, members of the epidermal growth factor receptor (EGFR) family, are known that lie at the head of a complex, multi-layered signaltransduction network. The EGF signaling pathway is activated via EGF ligands binding the EGF receptor (Fig. 1). Downstream the activated receptor complex invokes several signaling branches like the RAS-RAF-MEK-ERK (MAPK) cascade that activates cell proliferation and the PI3K/AKT pathway that has a cell-survival, anti-apoptotic effect [7]. Signal propagation via the MAPK pathway is passed through the adapter molecules Grb and Sos activating RAS. Subsequently, the signal propagates via RAF and MEK to ERK. Finally, active ERK promotes downstream several components that are involved in the induction of cell proliferation. In parallel, the active EGF receptor also activates PI3K that converts phosphatidylinositol-(4,5)-bisphosphate, $\mathrm{PI}(4,5) \mathrm{P}_{2}$, into phosphatidylinositol-(3,4,5)-trisphosphate, $\mathrm{PI}(3,4,5) \mathrm{P}_{3}$, and finally results in an activation of AKT. Active AKT inhibits apoptosis and promotes cell survival genes. We assume that other receptor tyrosine kinases have also a low-level effect on both signaling branches that is implemented in the model as an additional independent receptor. Refer also to Ref. [8] for a more comprehensive description of an EGF signaling model.
Different mutations and alterations have been found that can convey cancer development, like mutations of oncogenes with dominant gain of function (GoF), of tumor suppressor genes with recessive loss of function (LoF), or gene over-expression (OE), e.g., due to gene amplification. Several examples of such mutations are present in the EGF signaling pathway. These include an over-expression or mutation of the EGF receptor (EGFR, $[9,10]$ ), constitutively activated oncogenes like RAS [11,12], RAF [13] and PI3K [14], and loss of function mutations as for the PTEN phosphatase [15].

In recent years several drugs became available that specifically target components of cancer related pathways. There are also several that inhibit components of EGF mediated signal transduction. One of those targets is EGFR for which already several specific inhibitors are available [16]. One of these is Cetuximab (Erbitux $\left.{ }^{\mathrm{TM}}\right)$, a chimeric monoclonal antibody against EGFR [17]. It binds to the extracellular ligand binding domain of the EGFR receptor preventing the binding of EGF and TGF- $\alpha$ [18]. Another drug that is targeting EGFR directly is Erlotinib (Tarceva ${ }^{\mathrm{TM}}$ ). Erlotinib is binding the tyrosine kinase domain of EGFR [19]. Other drugs can specifically inhibit components of the PI3K/AKT branch, like PI-103 that inhibits PI3K [20], or the MAPK branch, for example U0126 that specifically inhibits MEK [21,22].

We generated a mathematical model of the EGF signaling pathway (cf. Fig. 1) using the PyBioS modeling and simulation system $[3,23]$. The individual reactions of the pathway are modeled by mass action kinetics. In addition to the native EGF signaling, also gain of function mutations for RAS, RAF, and PI3K, a loss of function mutation for PTEN, and an over-expression of EGFR were integrated and can be activated individually. Moreover, inhibitory drug effects for Erbitux, PI-103, Erlotinib, and U0126 can be introduced as indicated in Fig. 1.

\section{The Monte Carlo simulation strategy}

The reactions involved in the model consist of a small number of different reaction types such as synthesis reactions, product formation, and degradation reactions that are described by irreversible mass action kinetics $k \cdot \prod_{i=1}^{n} S_{i}$ where $k$ is a kinetic constant and $S_{i}$ is the concentration of the $i$ th substrate. Reversible reactions, as for example complex formation reactions, are described by the difference of two separate terms for the forward (complex association) and backward (complex dissociation) reaction each described by an irreversible mass action kinetic with a kinetic parameter $k_{\text {on }}$ for the association and a dissociation constant $K_{D}$. Synthesis and decay reactions have been introduced where appropriate. The rate laws of the model, which have been applied, are summarized in Table 1.

In our modeling approach we focus on predicting differences between different states of the same network, e.g., the biological network with or without inactivating different sets of drug targets (see Fig. 2). To compensate for the uncertainty in many of the parameters, the components of the parameter vector are chosen from appropriate probability distributions reflecting available knowledge. In the set of simulation runs described here, in particular, unknown kinetic parameters have been sampled from a log-normal distribution with mean $\mu=2.5$ and standard deviation $\sigma=0.5$. Each parameter vector and each vector of input values is used to model both the normal 'control' state as well as the 'treated' state, e.g., corresponding to an inhibition experiment. Thus, differences of the two states are due to changes in only one parameter or a small set of parameters. For simulation of the different inhibition experiments the model components representing drugs were initialized with fixed concentrations (U0126 $=1.0$, PI$103=1.0$, Erbitux =100.0, Erlotinib =1000.0; arbitrary units, a.u. . 


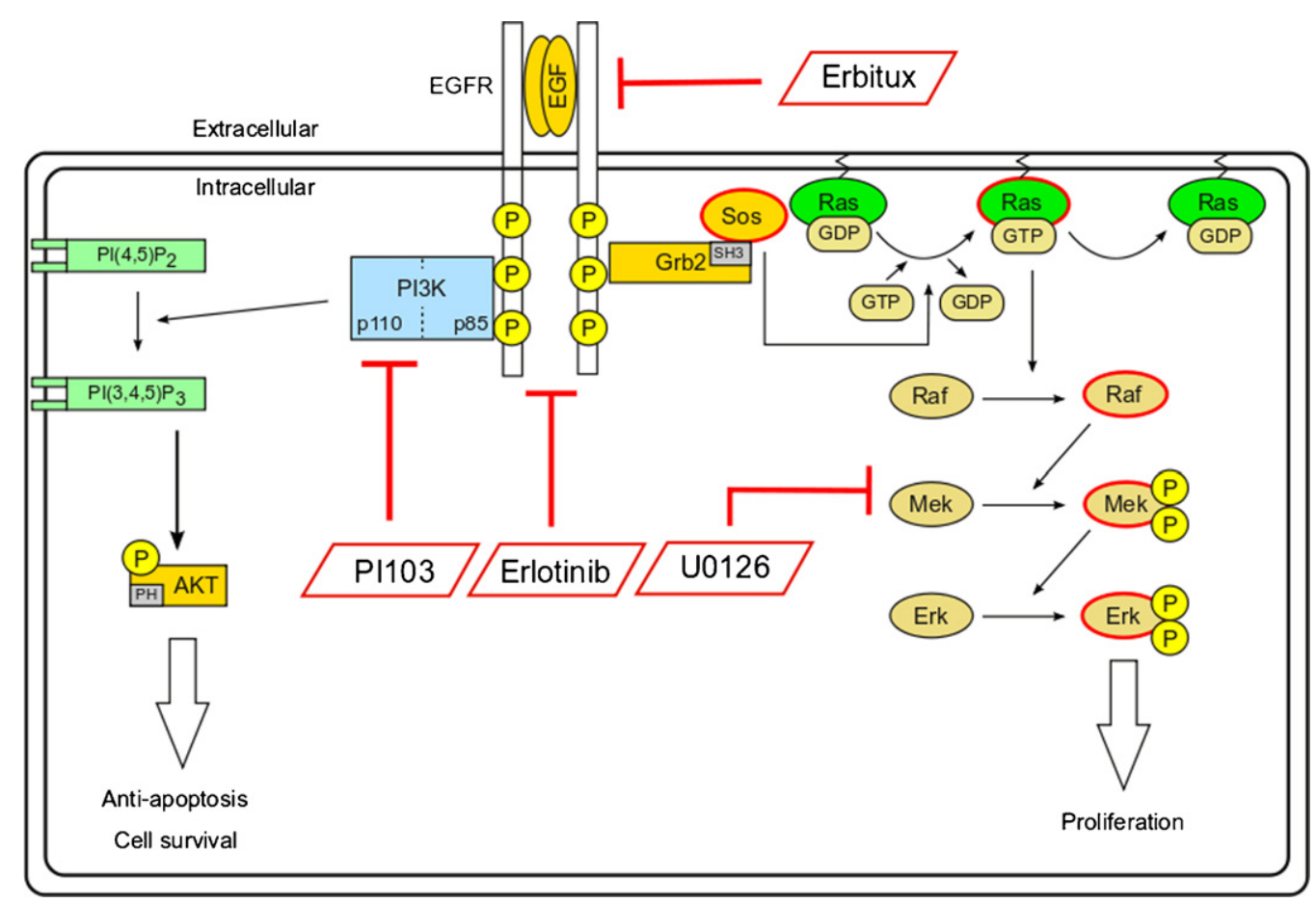

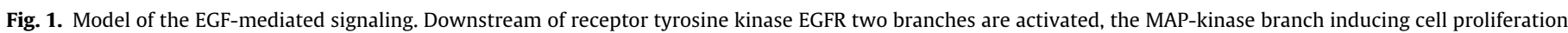
and the branch of PI3K/AKT inducing cell survival.

Control and treatment simulations were repeated 1000 times with different parameter vectors until steady state levels of all components were approximately reached within the simulation interval $[0 ; 20,000]$ (a.u.). The difference in model behavior between the 'treated' and the 'untreated' state is analyzed by comparing the steady state concentrations for each individual model component. Differences in the steady state values of the two states for the model components are analyzed by computing the geometric mean of the ratios of each individual pair (treatment vs. control) of Monte Carlo simulations. For the identification of significant changes of a model component between control and treatment the log2-ratios of relevant model components can be plotted into a heat map (see Section 4).

\section{Results}

In our approach we focus on predicting differences between different states of the same network, e.g., the biological network with or without structural alterations such as a mutation or a drug response. To compensate for the uncertainty in many of the parameters, the components of the parameter vector are chosen from an appropriate probability distribution as described above. In the following we apply the proposed Monte Carlo-based simulation approach on the EGF signaling model. In our analysis we compare the unstimulated signaling pathway with a perturbation such as an external stimulus by EGF, a mutation or a drug or combinations of those. Changes in the system state are judged by looking at certain readout components of the model. As a readout we use the cell survival (reflected by the amount of active AKT) or cell proliferation state (reflected by the amount of active ERK), each indicating the activity of the two signaling branches downstream of active EGFR (Fig. 1). Alternatively, one can look at the state of individual model components.

\subsection{Modeling the control and mutated states}

In a proof of principle in silico experiment we tested the effect of an extracellular EGF stimulus on the activity of the pathway. Therefore, the initial concentration of EGF was set to 10 a.u. in the case of stimulation and 0.1 a.u. for the unstimulated case. In addition, independent of the stimulation by EGF a constant stimulation by the additional growth factor ( 0.75 a.u.) was assumed. This results in a basal activity of the pathway in the control state. The results of 1000 Monte Carlo simulations both for control and

Table 1

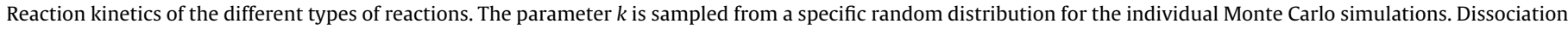
and inhibition constants are $k_{D}=k_{D I}=1$.

\begin{tabular}{|c|c|c|}
\hline Reaction & Biochemical & Kinetic rate law \\
\hline Synthesis & $\rightarrow \mathrm{S} 1$ & $v=k$ \\
\hline With gene & $\rightarrow \mathrm{S} 1$ & $v=k \times[G]$ \\
\hline Product formation & $\mathrm{S} 1 \rightarrow \mathrm{S} 2$ & $v=k \times[\mathrm{S} 1]$ \\
\hline With enzyme & $\mathrm{S} 1 \rightarrow \mathrm{S} 2$ & $v=k \times[\mathrm{S} 1] \times[\mathrm{E}]$ \\
\hline With inhibitor & $\mathrm{S} 1 \rightarrow \mathrm{S} 2$ & $v=k \times[\mathrm{S} 1] /\left(1 / k_{D \mathrm{I}} \times[\mathrm{I}]\right)$ \\
\hline With enzyme and inhibitor & $\mathrm{S} 1 \rightarrow \mathrm{S} 2$ & $v=k \times[\mathrm{S} 1] \times[\mathrm{E}] /\left(1 / k_{D \mathrm{I}} \times[\mathrm{I}]\right)$ \\
\hline Complex formation & $\mathrm{S} 1+\mathrm{S} 2 \leftrightarrow \mathrm{S} 1: \mathrm{S} 2$ & $v=k \times[\mathrm{S} 1] \times[\mathrm{S} 2]-k \times k_{D} \times[\mathrm{S} 1: \mathrm{S} 2]$ \\
\hline With inhibitor & $\mathrm{S} 1+\mathrm{S} 2 \leftrightarrow \mathrm{S} 1: \mathrm{S} 2$ & $v=k \times[\mathrm{S} 1] \times[\mathrm{S} 2] /\left(1 / k_{D \mathrm{I}} \times[\mathrm{I}]\right)-k \times k_{D} \times[\mathrm{S} 1: \mathrm{S} 2]$ \\
\hline Degradation & $\mathrm{S} 2 \rightarrow$ & $v=k \times[\mathrm{S} 2]$ \\
\hline
\end{tabular}



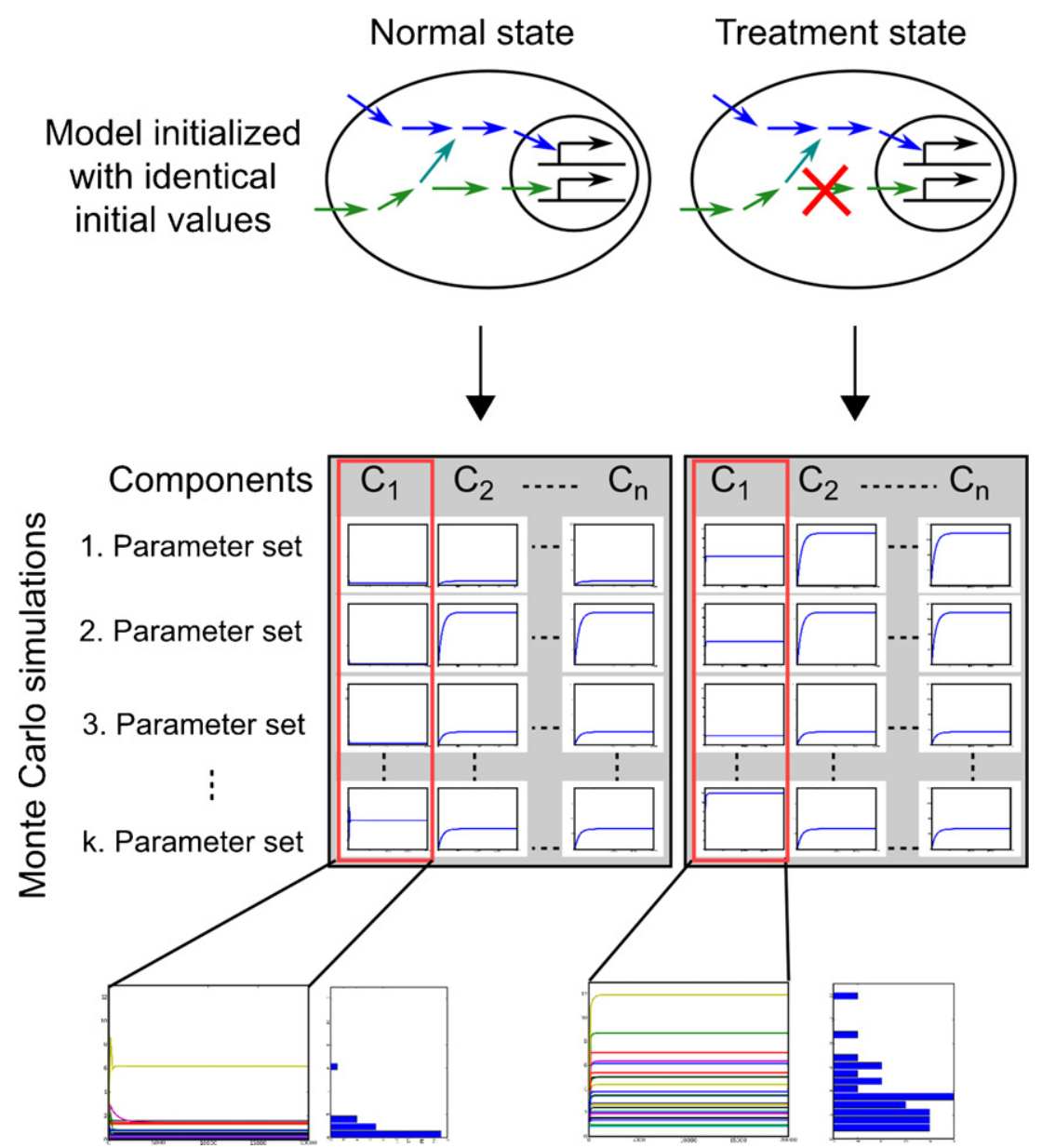

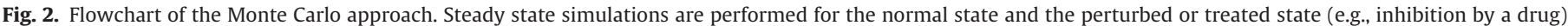

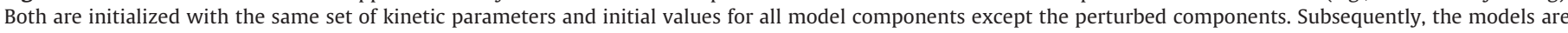

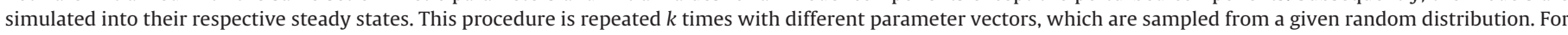

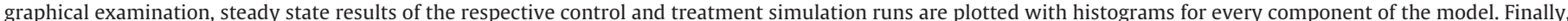

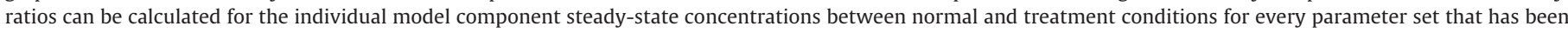

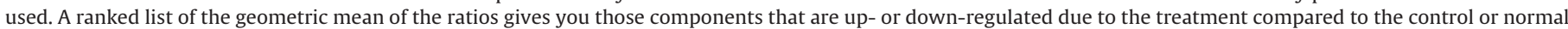
state.

treatment are depicted in Fig. 3. Both, cell survival and proliferation are up-regulated due to EGF stimulation (see Fig. 3 upper panel). This observation is in line with the expected biological behavior.

In a second experiment we tested the effect of a mutation of the oncogene RAF. Therefore, we assume that RAF has a gain of function mutation due to which RAF is constitutively active (see Fig. 3 lower panel). In this case, cell proliferation is increased in $89.8 \%$ of all Monte Carlo simulations compared to the unstimulated case. Only in $10.2 \%$ of the simulations a decrease in cell proliferation is observed. In contrast, we do not see any changes in the activity of the PI3K/AKT branch as indicated by cell survival. These qualitative results are in line with the expectations and demonstrate the applicability of the proposed approach.

\subsection{Analysis of mutation and drug effects on the state of the model}

In a second step, we used the Monte Carlo strategy to test different perturbations of the system, such as mutation or drug effects. Therefore, we have simulated different kinds of alterations of the EGF pathway that have been found to be related with cancer. In this analysis we focus on the oncogenes RAS, RAF, and PI3K with dominant gain of function mutations (GoF), the tumor suppressor gene PTEN with a recessive loss of function mutation (LoF), and an over-expression (OE) of the receptor EGFR. In addition we studied also the effect of targeted drugs on certain mutation patterns. Fig. 4 depicts the experiment design and the results of the simulations visualized in a heat map of the ratios of specific model components. As a reference for the computation of the ratios we used an unstimulated state (low-level constant stimulation by EGF ( 0.1 a.u.) and a constant stimulation by other growth factors (0.75 a.u.) via an EGFR-independent receptor tyrosine kinase.

Stimulation of the pathway by EGF (fixed concentration of 10.0 a.u.) results in an increase of cell survival and proliferation (Fig. 4, row 3). A similar effect can also be achieved by an EGFR OE or GoF mutation (rows 1 and 2). However, a GoF mutation in PI3K or a LoF mutation in PTEN results only in an increased activity of the PI3K/AKT signaling (rows 4 and 5), while a GoF mutation either in RAF or RAS only effects the MAPK signaling (rows 6 and 7).

Furthermore, we have analyzed the effects of targeted drugs in combination with certain mutation patterns. EGF stimulation can efficiently be blocked by Erbitux and Erlotinib (rows 10 and 13). This holds true also for an OE or GoF of EGFR, except for an Erbitux treatment in case of an EGFR GoF. Similarly, mutations in PI3K and PTEN or RAF and RAS cannot be impeded by either Erbitux or Erlotinib and cause activity of the respective branches of the pathway (rows 
Proliferation
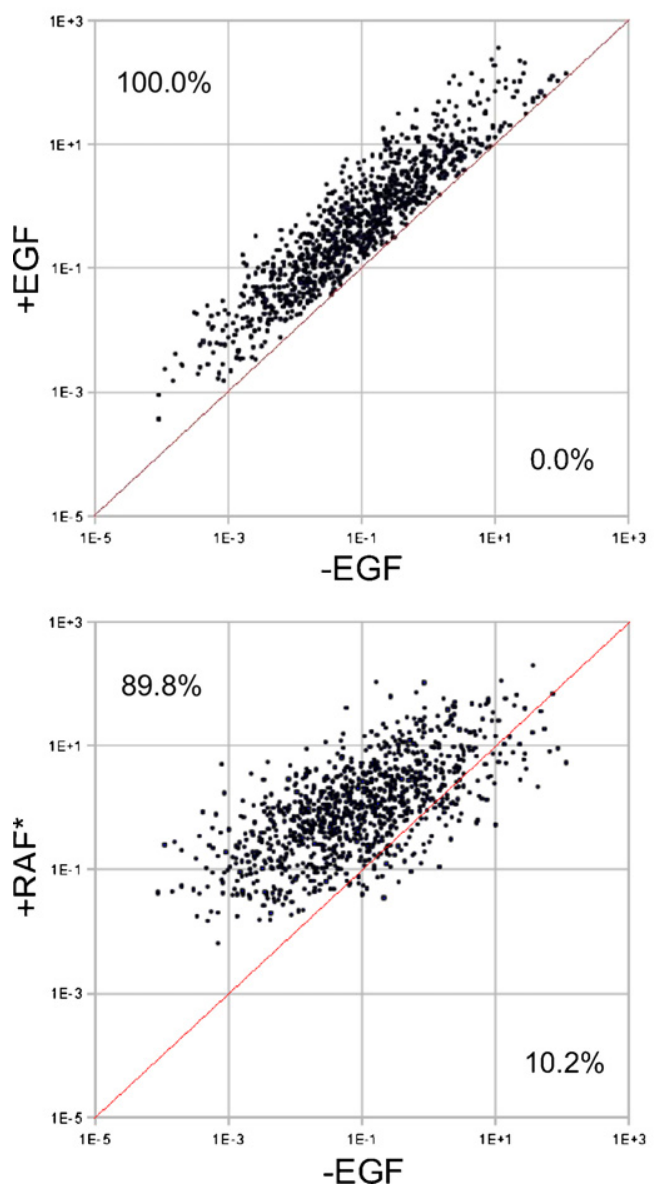

Cell Survival
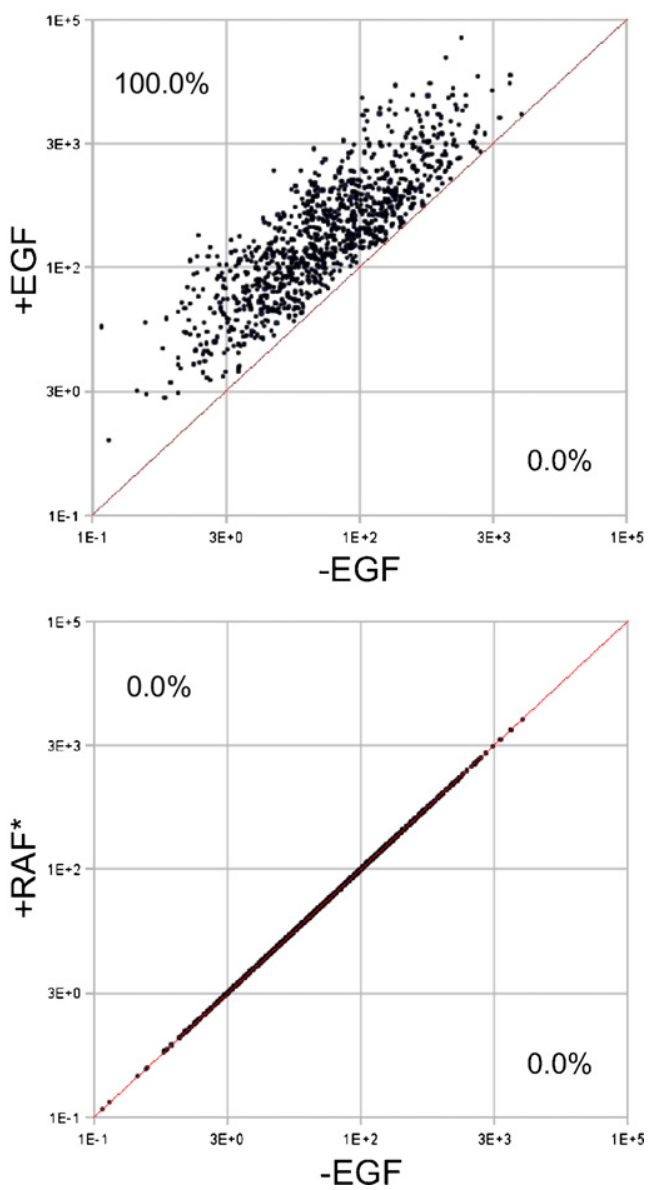

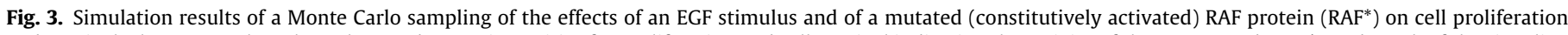

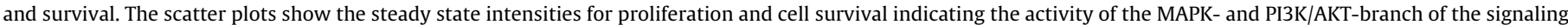

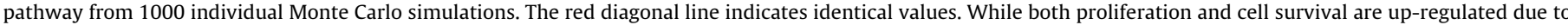

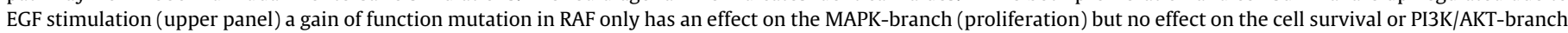
(lower panel).

14-17). In such a case, a component downstream of the mutated protein might be an appropriate drug target. Since cancer cells usually acquire multiple critical mutations in essential pathways to establish their phenotypes, it looks obvious that a combined targeted therapy might be essential to escape from the disease state. Hence, we have analyzed drug combinations to circumvent such a case. Our analysis demonstrates that any combination of an EGFR OE with a single mutation in one of the oncogenes or the tumor suppressor gene leading to induced cell survival and proliferation can be compensated by an appropriate drug combination (rows 18-27).

The presented simulation results are supported by evidence from the literature. As Pao et al. [24] have shown, tumors with specific KRAS mutations are associated with a lack of response to the kinase inhibitors Gefitinib or Erlotinib. BRAF, another component of the MAPK signal transduction, is also subject to a common activating mutation (V600E) in colorectal cancer and its response to Erbitux requires wild-type BRAF [25]. Also PIK3CA mutation or PTEN expression status predicts resistance of colon cancer cells to the epidermal growth factor receptor inhibitor cetuximab (Erbitux) [26]. Hence, Perrone et al. [27] suggest to inhibit molecules belonging to the downstream signaling of activated EGFR. As described above such results are also predicted by our simulations (Fig. 4). For instance, an over-expression of EGFR results in an increase of cell survival and proliferation (Fig. 5a vs. b). This can be reverted with an EGFR inhibitor such as Erbitux unless there is not any critical mutation in the downstream signaling (Fig. 5c). But a GoF mutation in an oncogene (e.g., $\mathrm{RAF}^{*}$ ) cannot be compensated and hence the therapy efficacy fades (Fig. 5d).

\section{Discussion and outlook}

Cancer is a complex disease and it has been found that multiple genetic changes are necessary before a cell can acquire tumorigenic competence, e.g., rodent cells require at least two genetic changes to acquire tumorigenic competence and their human counterparts are more difficult to transform [28]. Thus, several regulatory circuits must be disrupted in order for a cell to become cancerous. Hanahan and Weinberg [4] describe six essential alterations in cell physiology that collectively dictate malignant growth: selfsufficiency in growth signals, insensitivity to growth-inhibitory (antigrowth) signals, evasion of programmed cell death (apoptosis), limitless replicative potential, sustained angiogenesis, and tissue invasion and metastasis. Furthermore, Hanahan and Weinberg [5] also added reprogramming of energy metabolism and evading immune destruction. Hence, to understand a complex disease, such as cancer, we need to apply a systems biology approach integrating all relevant components, interactions, and pathways of the cellular reaction network and its effects on cell physiology. This leads to the analysis of large computational models. 


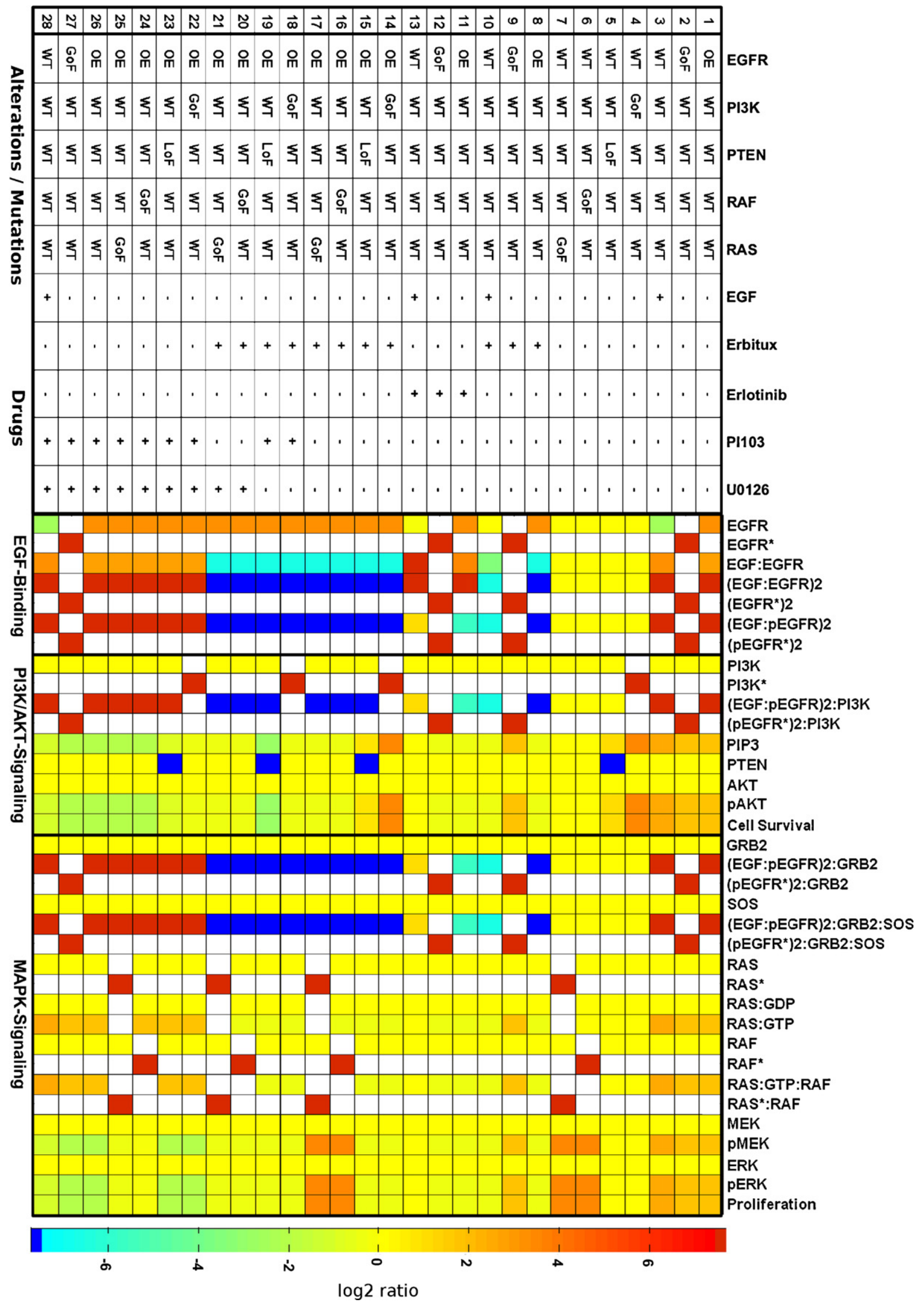

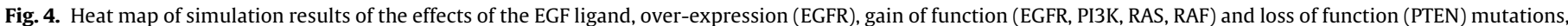

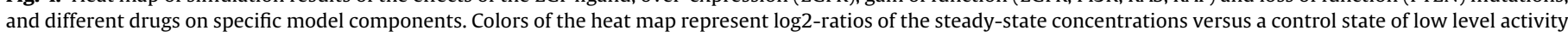
in cell survival and proliferation. 
(a)

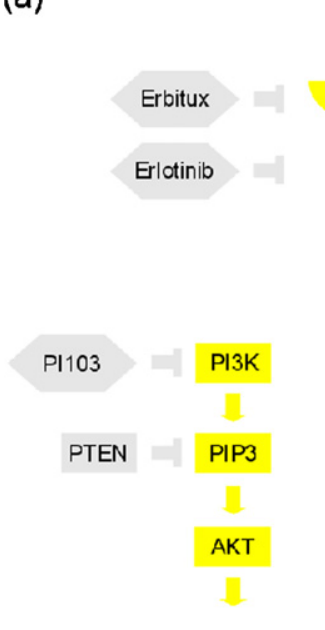

Cell Survival
EGF

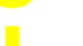

(b)

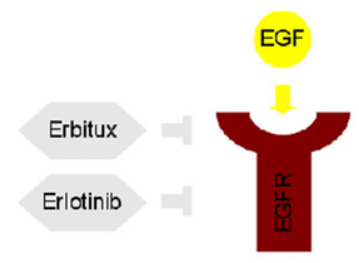

(c)

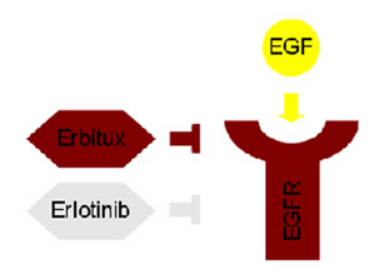

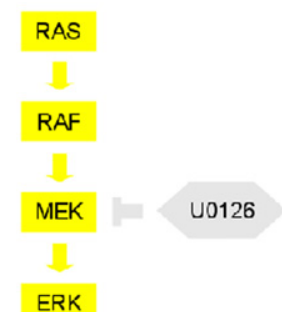

Proliferation

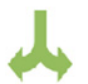

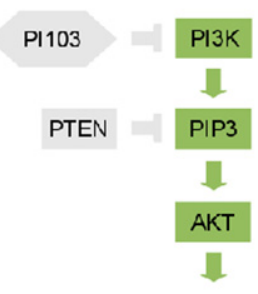

Cell Survival

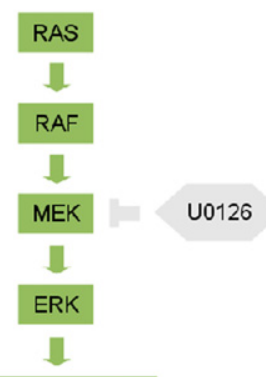

Proliferation
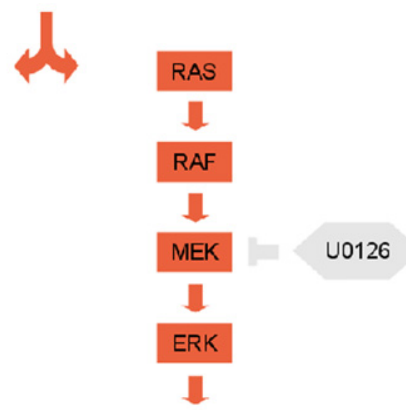

\section{Cell Survival Proliferation}

(d)
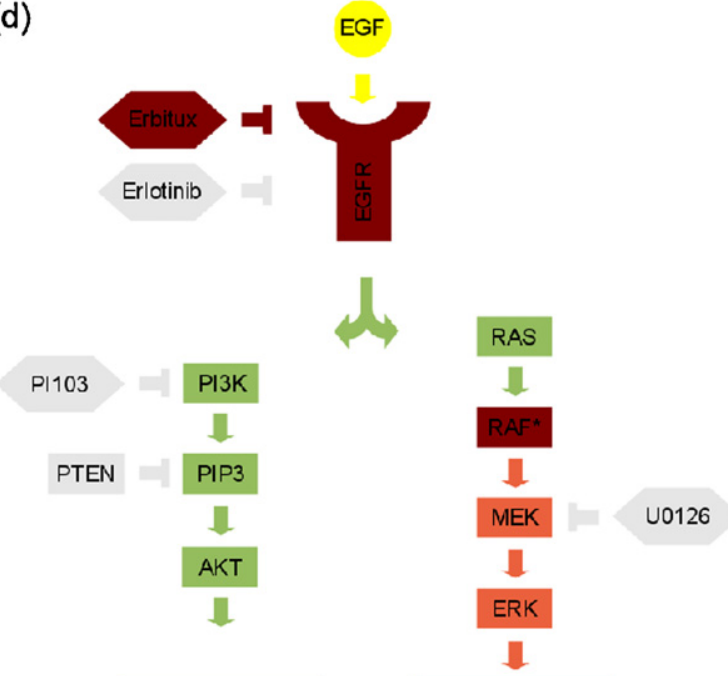

Cell Survival

Proliferation

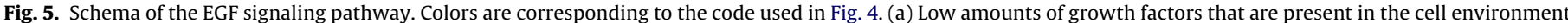

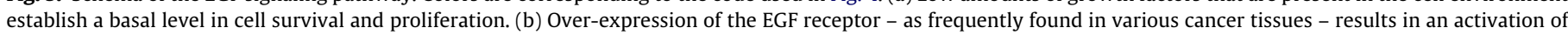

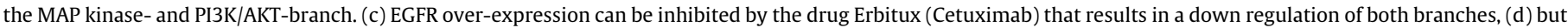
this cannot circumvent an increase in proliferation due to a downstream located mutation, e.g., in RAF*.

In this article we describe a strategy for deriving predictions from large networks coping with the fact that knowledge about the kinetic parameters is limited. By incorporating all known facts into the models and sampling the parameters from appropriate random distributions reflecting all available information or the lack of it, we can take advantage of this information, while handling the uncertainty inherent in any attempts to derive predictive models of biological networks of sufficient complexity to represent, e.g., complex disease states.

While predictions on a broad level (up and down regulation of model components with respect to different states) can often be generated in the absence of many model parameters, our approach is not restricted to that situation. The Monte Carlo approach can also be applied if different kinetic parameters are known to a certain extent and, thus, it will ultimately benefit from progress in the experimental approaches to measure such kinetic parameters.

Application of next generation sequencing of individual patients, combined with the development of systems that are able to model the disease process, can help to improve the understanding of the consequences of cancer related mutations on a molecular pathway level and their functional effects on the cellular and organism level. The analysis described here gives a first impression of the power of predictive models for the identification of new drug targets, as well as the improved targeting of therapies which are already available. The proposed modeling approach is a useful tool to study molecular interaction networks, such as signal transduction pathways, in silico to identify and formulate new hypothesis. However, qualitative or semi-quantitative predictions as provided by this modeling approach, have to be validated by appropriate in vitro or in vivo models. For instance, predicted drug effects have to be tested, e.g., in patient-specific cell lines or xenograft models, but finally, at least for approved drugs, the in silico predictions can also help the medical doctor to choose between alternative therapies.

The presented example of the EGF pathway is only a small piece of all the cellular signaling pathways that are involved in 
cancer development and progression [4,5]. However, to incorporate further factors such as hypoxia, crosstalk with other signal transduction pathways, redundancy of pathways and in this context also genomic instability leading to adaptations and acquired resistance, the underlying model has to be significantly extended with all cancer-related signaling pathways. Additional extensions of the model will also be necessary to cover other relevant factors, such as drug uptake or pharmacokinetics.

\section{Conflict of interest}

All authors declare that there are no conflicts of interest.

\section{Acknowledgments}

The work was funded by the German Ministry of Science and Education under its NGFN-Plus program (MUTANOM; 01GS08105) and its MedSys program (MoGLI, 0315394A), by the European Commission under its Framework 6 project carcinoGENOMICS (LSHB-CT-2006-037712) and by the Max Planck Society.

Study sponsors were not involved in the study design; collection, analysis and interpretation of data; the writing of the manuscript; the decision to submit the manuscript for publication.

\section{References}

[1] H. Kitano, Computational systems biology, Nature 420 (2002) 206-210.

[2] E. Klipp, R. Herwig, A. Kowald, C. Wierling, H. Lehrach, Systems Biology in Practice: Concepts, Implementation and Application, Weinheim, Wiley-VCH, 2005.

[3] E. Klipp, W. Liebermeister, C. Wierling, A. Kowald, H. Lehrach, R. Herwig, Systems Biology-A Textbook, Wiley-VCH, Weinheim, 2009.

[4] D. Hanahan, R.A. Weinberg, The hallmarks of cancer, Cell 100 (2000) 57-70.

[5] D. Hanahan, R.A. Weinberg, Hallmarks of cancer: the next generation, Cell 144 (2011) 646-674.

[6] Y. Yarden, The EGFR family and its ligands in human cancer: signalling mechanisms and therapeutic opportunities, Eur. J. Cancer 37 (2001) S3-S8.

[7] M. Osaki, M. Oshimura, H. Ito, PI3K-Akt pathway: its functions and alterations in human cancer, Apoptosis 9 (2004) 667-676.

[8] K. Oda, Y. Matsuoka, A. Funahashi, H. Kitano, A comprehensive pathway map of epidermal growth factor receptor signaling, Mol. Syst. Biol. 1 (2005) 2005.0010

[9] H.K. Shu, R.J. Pelley, H.J. Kung, Tissue-specific transformation by epidermal growth factor receptor: a single point mutation within the ATP-binding pocket of the erbB product increases its intrinsic kinase activity and activates its sarcomagenic potential, Proc. Natl. Acad. Sci. U.S.A. 87 (1990) 9103-9107.

[10] R.I. Nicholson, J.M. Gee, M.E. Harper, EGFR and cancer prognosis, Eur. J. Cancer 37 (Suppl. 4) (2001) S9-S15.

[11] M. Malumbres, M. Barbacid, RAS oncogenes: the first 30 years, Nat. Rev. Cancer 3 (2003) 459-465.

[12] E.F. Pai, W. Kabsch, U. Krengel, K.C. Holmes, J. John, A. Wittinghofer, Structure of the guanine-nucleotide-binding domain of the Ha-ras oncogene product p21 in the triphosphate conformation, Nature 341 (1989) 209-214.
[13] H. Davies, G.R. Bignell, C. Cox, P. Stephens, S. Edkins, S. Clegg, J. Teague, H Woffendin, M.J. Garnett, W. Bottomley, et al., Mutations of the BRAF gene in human cancer, Nature 417 (2002) 949-954.

[14] I. Vivanco, C.L. Saywers, The phosphatidylinositol 3-kinase AKT pathway in human cancer, Nat. Rev. Cancer 2 (2002) 489-501.

[15] P.A. Steck, M.A. Pershouse, S.A. Jasser, W.K. Yung, H. Lin, A.H. Ligon, L.A. Lang ford, M.L. Baumgard, T. Hattier, T. Davis, C. Frye, R. Hu, B. Swedlund, D.H. Teng, S.V. Tavtigian, Identification of a candidate tumour suppressor gene, MMAC1, at chromosome 10q23.3 that is mutated in multiple advanced cancers, Nat. Genet. 15 (1997) 356-362.

[16] S.M. Huang, P.M. Harari, Epidermal growth factor receptor inhibition in cancer therapy: biology, rationale and preliminary clinical results, Invest. New Drugs 17 (1999) 259-269.

[17] J. Baselga The, EGFR as a target for anticancer therapy-focus on cetuximab, Eur. J. Cancer 37 (Suppl. 4) (2001) 16-22.

[18] Y. Humblet, Cetuximab: an IgG(1) monoclonal antibody for the treatment of epidermal growth factor receptor-expressing tumours, Expert Opin. Pharmacother. 5 (2004) 1621-1633.

[19] J.D. Moyer, E.G. Barbacci, K.K. Iwata, L. Arnold, B. Boman, et al., Induction of apoptosis and cell cycle arrest by CP-358,774, an inhibitor of epidermal growth factor receptor tyrosine kinase, Cancer Res. 57 (1997) 4838.

[20] Q.W. Fan, Z.A. Knight, D.D. Goldenberg, W. Yu, K.E. Mostov, D. Stokoe, K.M Shokat, W.A. Weiss, A dual PI3 kinase/mTOR inhibitor reveals emergent efficacy in glioma, Cancer Cell 9 (2006) 341-349.

[21] J.V. Duncia, J.B. Santella 3rd, C.A. Higley, W.J. Pitts, J. Wityak, W.E. Frietze, F.W. Rankin, J.H. Sun, R.A. Earl, A.C. Tabaka, C.A. Teleha, K.F. Blom, M.F. Favata, E.J. Manos, A.J. Daulerio, D.A. Stradley, K. Horiuchi, R.A. Copeland, P.A. Scherle, J.M. Trzaskos, R.L. Magolda, G.L. Trainor, R.R. Wexler, F.W. Hobbs, R.E. Olson, MEK inhibitors: the chemistry and biological activity of U0126, its analogs, and cyclization products, Bioorg. Med. Chem. Lett. 8 (1998) 2839-2844.

[22] M.F. Favata, K.Y. Horiuchi, E.J. Manos, A.J. Daulerio, D.A. Stradley, W.S. Feeser D.E. Van Dyk, W.J. Pitts, R.A. Earl, F. Hobbs, R.A. Copeland, R.L. Magolda, P.A. Scherle, J.M. Trzaskos, Identification of a novel inhibitor of mitogen-activated protein kinase kinase, J. Biol. Chem. 273 (1998) 18623-18632.

[23] C. Wierling, R. Herwig, H. Lehrach, Resources, standards and tools for systems biology, Brief. Funct. Genom. Proteom. 6 (2007) 240-251.

[24] W. Pao, T.Y. Wang, G.J. Riely, V.A. Miller, Q. Pan, M. Ladanyi, M.F. Zakowski, R.T. Heelan, M.G. Kris, H.E. Varmus, KRAS mutations and primary resistance of lung adenocarcinomas to gefitinib or erlotinib, PLoS Med. 2 (2005) e17.

[25] F. Di Nicolantonio, M. Martini, F. Molinari, A. Sartore-Bianchi, S. Arena, P. Saletti, S. De Dosso, L. Mazzucchelli, M. Frattini, S. Siena, A. Bardelli, Wild-type BRAF is required for response to panitumumab or cetuximab in metastatic colorectal cancer, J. Clin. Oncol. 26 (2008) 5705-5712.

[26] M. Jhawer, S. Goel, A.J. Wilson, C. Montagna, Y.H. Ling, D.S. Byun, S. Nasser, D. Arango, J. Shin, L. Klampfer, L.H. Augenlicht, R. Perez-Soler, J.M. Mariadason, PIK3CA mutation/PTEN expression status predicts response of colon cance cells to epidermal growth factor receptor inhibitor cetuximab, Cancer Res. 68 (2008) 1953-1961.

[27] F. Perrone, A. Lampis, M. Orsenigo, M. Di Bartolomeo, A. Gevorgyan, M. Losa, M. Frattini, C. Riva, S. Andreola, E. Bajetta, L. Bertario, E. Leo, M.A. Pierotti, S. Pilotti, PI3KCA/PTEN deregulation contributes to impaired responses to cetuximab in metastatic colorectal cancer patients, Ann. Oncol. 20 (2009) 84-90.

[28] W.C. Hahn, C.M. Counter, A.S. Lundberg, R.L. Beijersbergen, M.W. Brooks, R.A. Weinberg, Creation of human tumour cells with defined genetic elements, Nature 400 (1999) 464-468. 